\title{
Efectividad de la analgesia controlada por el paciente en el dolor agudo y crónico después de cirugía cardiaca: estudio prospectivo
} Effectiveness of patient-controlled analgesia in acute
and chronic pain after cardiac surgery: a prospective study

N. Esteve-Pérez ${ }^{1,2}$, J. Iborra-Escalona1, G. Gómez-Romero ${ }^{1,2}$, C. Sansaloni-Perelló ${ }^{1}$, A. M. Verger-Bennasar¹, S. Tejada-Gavela ${ }^{3}$, M. Riera-Sagrera ${ }^{4}$ y L. C. Mora-Fernández ${ }^{1,2,5}$

'Servicio de Anestesia, Reanimación y Terapia del Dolor. Hospital Universitario Son Espases. Palma de Mallorca, España. 2Instituto de Investigación Sanitaria de las Islas Baleares (IdISBA), Palma de Mallorca, España. ${ }^{3}$ Departamento de Biología de la Universidad de las Islas Baleares. CIBEROBN (Physiopathology of Obesity and Nutrition], Palma de Mallorca, España. ${ }^{4}$ Jefe de Sección de la Unidad de Cuidados Intensivos.

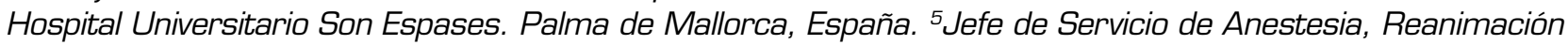
y Terapia del Dolor. Hospital Universitario Son Espases. Palma de Mallorca, España

\section{ABSTRACT}

Introduction: The intensity of postoperative moderate/ intense pain after cardiac surgery (CC), varies according to the different studies, from $45 \%$ to $85 \%$. There is no evidence about which is the optimal analgesic regimen in the postoperative period.

The main objective of this study is to evaluate the effectiveness of the application of a multimodal analgesic protocol, based on patient-controlled analgesia (PCA) with morphine, in patients undergoing cardiac surgery with extracorporeal circulation (ECC).

Patients and methods: Prospective observational study of all patients undergoing CS with ECC, during the first 3 days postoperatively. There were included 102 patients in two periods, first, in November 2016 with conventional analgesia and second, in January - February 2017 with PCA.

Results: The pain at rest was controlled (median numerical scale $<3$ ). An average of $27 \%$ of moderate and intense pain was recorded. There was no difference in pain intensity between patients with CA and those with PCA. The PCA group required less rescue analgesia in the first postoperative days (63 \% vs. $44 \% p=0.0487$ ). The incidence of Post-surgical Chronic Pain was $39 \%$

\section{RESUMEN}

Introducción: La intensidad del dolor postoperatorio moderado-intenso después de una cirugía cardiaca (CC) varía, según los estudios, de un 45 a un $85 \%$. No existe evidencia sobre cuál es la pauta analgésica óptima en el postoperatorio de estos pacientes.

El objetivo de este estudio es evaluar la efectividad de la aplicación de un protocolo analgésico multimodal, basado en la analgesia controlada por el paciente (PCA) con morfina, en pacientes sometidos a CC con circulación extracorpórea (CEC).

Pacientes y métodos: Estudio prospectivo de todos los pacientes sometidos a CC con CEC, durante los primeros 3 días del postoperatorio (DPO). Se incluyeron 102 pacientes en dos periodos, noviembre de 2016 , con analgesia convencional $(A C)$ y enero-febrero de 2017 con PCA.

Resultados: El dolor en reposo se mantuvo controlado (mediana escala numérica < 3). Se registró un $27 \%$ de pacientes con dolor moderado e intenso. No hubo diferencias en la intensidad del dolor entre los pacientes con AC y los de PCA. El grupo de PCA precisó menos analgesia de rescate (63 vs. $44 \%, p=0,0487$ ). La incidencia de dolor crónico postquirúrgico fue de un
Esteve-Pérez N, Iborra-Escalona J, Gómez-Romero G, Sansaloni-Perelló C, Verger-Bennasar AM, Tejada-Gavela S, Riera-Sagrera M, Mora-Fernández LC. Efectividad de la analgesia controlada por el paciente en el dolor agudo y crónico después de cirugía cardiaca: estudio prospectivo. Rev Soc Esp Dolor 2020;27(1):24-36
Recibido: 02-06-2019 Aceptado: 02-01-2020

Correspondencia: Neus Esteve-Pérez neus.esteve@ssib.es 
at three months, and $3 \%$ at one year. There was a correlation between preoperative pain and anxiety with the intensity of the dynamic pain ( $r=0.287, p=0.03$ ).

Conclusions: PCA with on-demand opioids and multimodal analgesia is an effective alternative after cardiac surgery. Good control of postoperative pain is obtained without increasing adverse effects, and requiring less rescue analgesia administered by the nursing staff.

Key words: Postoperative Analgesia, cardiac surgery, multimodal analgesia, patient controlled analgesia (PCA).

\section{INTRODUCCIÓN}

El control eficaz del dolor agudo postoperatorio (DAP) se ha convertido en una parte esencial de los cuidados perioperatorios y su adecuado tratamiento, junto a otros factores como la movilización y la nutrición pre$\mathrm{coz}$, se relacionan con la disminución de las complicaciones postoperatorias y de la estancia hospitalaria (1). La presencia de DAP se ha asociado a un aumento de la morbilidad y de los costes [2], a una disminución del bienestar del paciente y a un riesgo más elevado de desarrollar dolor crónico [3].

EI DAP tras cirugía cardiaca (CC) se describe como de moderado a intenso y con una repercusión fisiopatológica potencial importante. El máximo grado de dolor se percibe durante los dos primeros días del postoperatorio. Comienza a disminuir a partir del tercer día tras la cirugía y se considera leve a partir de la primera semana [4]. Se origina en diversos puntos anatómicos: esternotomía, retracción esternal y costal, pericardiotomía, disección de la arteria mamaria interna, de la vena safena, manipulación de la pleura parietal, inserción de tubos torácicos (mediastínicos y pleurales), canulación de vías venosas o arteriales y otros traumatismos musculoesqueléticos [5].

La prevalencia del DAP en CC varía mucho según los estudios. Navarro y cols. [6] describieron que un $45 \%$ de los pacientes presentaban dolor de moderado a intenso en las primeras 24 horas. Keawnantawat y cols. (7) registraron un $70 \%$ y Bjørnnes y cols. (8) un $85 \%$. Los estudios son muy heterogéneos, por lo que es difícil obtener conclusiones válidas. No obstante, los autores coinciden en que la incidencia de dolor moderado a intenso en la CC es elevada, y que probablemente se prescriban tratamientos analgésicos insuficientes. Por otro lado, no existe evidencia concluyente sobre cuál es la pauta analgésica óptima en el postoperatorio de estos pacientes (9).

El objetivo principal de este estudio es evaluar la efectividad de la aplicación de un protocolo analgésico multimodal, basado en la analgesia controlada por el paciente con morfina intravenosa, en pacientes sometidos a cirugía cardiaca con circulación extracorpórea (CEC), durante los 3 primeros días del postoperatorio.
$39 \%$ a los tres meses y un $3 \%$ al año. El dolor y la ansiedad preoperatorios se correlacionaron con la intensidad del dolor dinámico ( $r=0,287, p=0,03$ ).

Conclusiones: La PCA con opioides a demanda y analgesia multimodal es una alternativa efectiva después de la CC. Se obtiene un buen control del dolor postoperatorio sin incrementar los efectos adversos y precisando menos analgesia de rescate administrada por enfermería.

Palabras clave: Analgesia postoperatoria, cirugía cardiaca, analgesia multimodal, analgesia controlada por el paciente (PCA).
La intensidad del dolor se relaciona con las variables demográficas, la comorbilidad de los pacientes, los datos intra y postoperatorios y también con la presencia de dolor crónico postquirúrgico (DCPQ), al año de la intervención.

\section{MATERIAL Y MÉTODOS}

\section{Diseño}

Estudio prospectivo, observacional, mediante entrevista y revisión de la historia clínica, de todos los pacientes sometidos a CC con CEC, durante los 3 primeros días del postoperatorio, en la Unidad de Cuidados Intensivos (UCI) y en la planta de hospitalización. Se incluyen todos los pacientes antes y después de la implementación de un protocolo de analgesia multimodal perioperatoria, basada en la analgesia controlada por el paciente con morfina.

El estudio fue aprobado por el Comité de Ética en la Investigación de las Islas Baleares, (N. ${ }^{\circ}$ IB 3376/16 PI) y se siguieron las recomendaciones de la Declaración de Helsinki, referente a la investigación en biomedicina. Todos los pacientes fueron informados sobre el protocolo del estudio antes de firmar el consentimiento informado específico.

\section{Población}

Se incluyeron todos los pacientes en dos periodos consecutivos, en noviembre de 2016 con analgesia convencional (grupo AC), y en enero-febrero $2017 \mathrm{con}$ analgesia controlada por el paciente (grupo PCA).

Se incluyeron todos los pacientes mayores de 18 años, que aceptaron participar en el estudio y que firmaron el consentimiento informado, programados para CC con CEC y posterior ingreso en UCl. Se excluyeron los pacientes menores de edad, pacientes que rechazaron participar en el estudio, pacientes con déficit cognitivo o imposibilidad de comunicación verbal o pacientes cuya conexión a ventilación mecánica invasiva se prolongó más de 24 horas posteriores a la cirugía. 


\section{Procedimientos}

Los pacientes fueron sometidos a cirugía coronaria, cirugía valvular o ambas, programada o urgente. El protocolo quirúrgico incluyó esternotomía media, o toracotomía o abordaje mínimamente invasivo, circulación extracorpórea con moderada hipotermia $\left(32{ }^{\circ} \mathrm{C}\right.$ ), y disección de la arteria mamaria interna, de la vena safena o de ambas.

El protocolo anestésico incluyó la monitorización hemodinámica invasiva, ventilatoria y de la profundidad anestésica. Se aplicó la oximetría cerebral no invasiva y la ecocardiografía transesofágica según indicación del anestesiólogo. La inducción se realizó con midazolam, fentanilo y propofol. El mantenimiento con sevofurano o propofol, según preferencias del anestesiólogo. La analgesia intraoperatoria se realizó con bolos a demanda de fentanilo (dosis total 10-15 $\mathrm{g} \mathrm{kg} \mathrm{k}^{-1}$ ) o perfusión de remifentanilo [dosis < 0,2 $\mathrm{gg} \mathrm{kg}^{-1} \mathrm{~min}^{-1}$ ]. Para la relajación muscular se utilizó rocuronio o cisatracurio. Se aplicó analgesia preventiva como parte del protocolo analgésico a los pacientes del grupo PCA, 30 minutos antes de la salida de quirófano, con dexketoprofeno (50 mg) o metamizol ( 2 g), paracetamol (1 g), dexametasona (8 mg) y tramadol (100 mg) o morfina (5 mg). Los pacientes se trasladaron a UCl, siendo extubados cuando cumplieron criterios de estabilidad hemodinámica y respiratoria.

En el grupo AC, tras la extubación, se pautó dexketoprofeno (50 mg/8 h) o metamizol ( $2 \mathrm{~g} / 8 \mathrm{~h}$ ) y paracetamol ( $1 \mathrm{~g} / 6 \mathrm{~h}$ ) con $5 \mathrm{mg}$ intravenosos de morfina, como analgesia de rescate, cuando la EN $>4$.

\section{Intervención}

En el grupo PCA, tras la extubación, se pautó una bomba de PCA con morfina ( $1 \mathrm{mg} / 1 \mathrm{ml}$ ), a demanda, con bolos de $1 \mathrm{mg}$, tiempo de cierre de 10 min y un máximo de 6 bolos/hora. Además se administró dexketoprofeno (50 mg/8 h) o metamizol ( $2 \mathrm{~g} / 8 \mathrm{~h}$ ) y paracetamol ( $1 \mathrm{~g} /$ $6 \mathrm{~h}$ ) y bolos de $5 \mathrm{mg}$ intravenosos de morfina, como analgesia de rescate, cuando la EN > 4. La bomba se retiró a las 48 horas, si el dolor estaba controlado (EN < 4) y se consumieron menos de 10 bolos /24 h.

\section{Registro de datos}

Los pacientes incluidos en el estudio respondieron a tres encuestas diferentes: una encuesta preoperatoria, una postoperatoria del DAP en los tres primeros días del postoperatorio, y una encuesta telefónica postoperatoria del DCPQ que se cumplimentó a los 3, 6 y 12 meses del postoperatorio. Además, se recogieron los datos de la historia clínica de los pacientes.

Las encuestas a los pacientes fueron realizadas por tres miembros del equipo del estudio, entrenados en la evaluación del dolor postoperatorio.

La encuesta preoperatoria, adaptada del Brief Pain Inventory (10) (BPI) (Anexo 1), explora la presencia de dolor, ansiedad, depresión y catastrofismo.

La encuesta postoperatoria del DAP (Anexo 2) se basa en el cuestionario adaptado de la Sociedad Americana del Dolor [11]. Se registró la intensidad del dolor en reposo y en movimiento medida con la escala numérica verbal (EN O a 10, 0 = no dolor, 10 = máximo dolor). El grado de dolor fue registrado en el momento de la entrevista (dolor observado), así como la máxima intensidad que refirió el paciente en el postoperatorio [dolor máximo percibido]. Los pacientes se clasificaron según el grado de dolor: ausencia de dolor o dolor leve $(E N<3$ ), dolor moderado (EN 4-6), dolor intenso (EN > 6) y cualquier grado de dolor ( $E N>4$ ). Se registró también la localización del dolor en tres áreas (la esternotomía, la salida de los drenajes y dolor musculoesquelético), la necesidad de analgesia de rescate, los posibles efectos adversos de los analgésicos y el grado de descanso nocturno.

Finalmente, se preguntó a los pacientes acerca de las expectativas y la realidad sobre el dolor percibido, y la satisfacción sobre el tratamiento analgésico recibido.

La encuesta postoperatoria del DCPQ (Anexo 3) se basa en el test de Lattinen (12) sobre el dolor crónico. Se repitieron las preguntas sobre el catastrofismo realizadas en la encuesta preoperatoria.

\section{Variables}

Se registraron las variables demográficas, el ASA (American Society of Anesthesiologists), la escala NYHA (New York Heart Association) y el EUROSCORE (European System for Cardiac Operative Risk Evaluation] versión I y II.

Como variables de comorbilidad se definieron, Diabetes mellitus, riesgo insuficiencia renal [creatinina > 1,2 ), dolor crónico (dolor de más de 3 meses de duración en cualquier localización, con consumo crónico de analgésicos], síndrome ansioso-depresivo (consumo crónico de benzodiacepinas o psicótropos], cirugía mayor previa, cirugía cardiaca previa y consumo de opioides o drogas.

Las variables perioperatorias registradas fueron el tipo de cirugía cardiaca, el tipo de abordaje quirúrgico, los tipos de drenajes, la duración de la CCA y la CEC, el manejo anestésico de mantenimiento y analgesia (propofol, sevofurane, remifentanilo, fentanilo o morfina), la administración de analgesia preventiva, los analgésicos pautados en los 3 primeros días del postoperatorio (intravenosos u orales] y la analgesia de rescate.

Las variables de la encuesta postoperatoria del $D C P Q$ que se registraron fueron la intensidad y frecuencia del dolor (de $\mathrm{O}$ a 4), el consumo de analgésicos (de 0 a 4), la capacidad funcional (de 0 a 4) y la calidad del sueño (de O a 4).

\section{Tamaño de la muestra}

Revisada la literatura sobre la efectividad analgésica, esta se definió como una reducción del $20 \%$ en la puntuación de la EN en el grupo PCA (13). Con un poder de 0,8 y un nivel de significación del 0,05, se necesitarían un mínimo de 26 pacientes en cada grupo.

\section{Análisis estadístico}

Se llevó a cabo con el paquete estadístico SPSS, versión 24.0. Para describir las variables cualitativas 
se utilizaron las frecuencias absolutas y los porcentajes. La descripción de las variables cuantitativas se realizó mediante valores medios y desviación estándar o medianas y rango intercuartil ( $R \mid Q$ Q1-Q3). Para comparar las características de los pacientes dependiendo del tipo de pauta analgésica y la identificación de variables relacionadas con el grado de dolor se aplicaron los test $\cup$ de Mann-Whitney y Chi cuadrado o la prueba exacta de Fisher para variables cuantitativas y cualitativas, respectivamente. Se consideraron estadísticamente significativos valores de $p<0,05$.

\section{RESULTADOS}

Se evaluaron 102 pacientes, 52 con tratamiento analgésico convencional (AC) y 50 con PCA. En la Figura 1 se muestra el diagrama de flujo con el protocolo del estudio y los pacientes excluidos. En las Tablas I y II se muestran los datos demográficos, de comorbilidad, y los datos intraoperatorios. No hubo diferencias en los datos demográficos, la comorbilidad, las escalas de riesgo o el tipo de intervención quirúrgica, entre los dos grupos. No se registró ningún fallecimiento.

El dolor en reposo se mantuvo controlado (mediana EN < 3), mientras que se registró dolor de moderado a intenso en movimiento, desde la extubación (mediana EN 5, rango intercuartil [RIQ] 2-8) hasta el tercer DPO, (mediana EN 5, RIQ 3-7). Se registró una gran variabilidad interindividual, con rangos máximo y mínimo de 10 y 0 respectivamente. El porcentaje de pacientes con dolor controlado en reposo fue del $68 \%$ en la extubación y del $85 \%$ en el tercer DPO. El dolor moderado e intenso en reposo fue del $27 \%$, con una evolución desde un $19 \%$ moderado y $14 \%$ intenso en la extu- bación hasta un $13 \%$ moderado y $4 \%$ intenso en el tercer DPO. El dolor máximo referido por el paciente en reposo se mantuvo por debajo de EN 3, con un 44 $\%$ de los pacientes con dolor moderado el primer día postoperatorio, y un $25 \%$ al tercer día.

En la Figura 2 mostramos la evolución del dolor observado desde la extubación hasta el tercer día postoperatorio. En la Figura 3, se muestra la evolución del dolor máximo referido por el paciente.

La localización del dolor en la extubación y a lo largo de los primeros 3 días fue de un $89 \%$ en la esternotomía, un $9 \%$ de origen musculoesquelético y un $2 \%$ en los drenajes.

No hubo diferencias en la intensidad del dolor entre los pacientes con AC y los de PCA, ni en reposo ni en movimiento, en los tres DPO (Figura 4).

Precisaron analgesia de rescate el $54 \%$ de los pacientes en la extubación, el $54 \%$ en el primer DPO y el $10 \%$ y el $1 \%$ en el 2 y 3 DPO, respectivamente. El grupo de PCA precisó menos analgesia de rescate en el primer DPO que el grupo con analgesia convencional, (63 \% vs. $44 \%, p=0,0487$ ) y también menos milígramos de morfina, [ $1,9 \mathrm{mg} /$ paciente vs. $4,5 \mathrm{mg} /$ paciente, $p=0,0461$ ). La conversión de la analgesia a vía oral se realizó al tercer DPO en un $92 \%$ de los pacientes del grupo PCA y en un $46 \%$ de los pacientes del grupo de $A C(p<0,001)$.

Con respecto al descanso nocturno, no pudieron conciliar el sueño un $40 \%$ de los pacientes el primer DPO y un $34 \%$ y $25 \%$ en el 2 y 3 DPO, respectivamente. Sin diferencias entre ambos grupos.

Un $35 \%$ de los pacientes refirieron haber tenido más dolor del que esperaban, sin diferencias entre los dos grupos de pacientes, (PCA $34 \%$ vs. AC $36 \%$, $p=0,788$ ]. El $95 \%$ mostró su satisfacción con el

\begin{tabular}{|c|c|}
\hline 113 pacientes & \\
\hline $\begin{array}{l}\text { Encuesta preoperatoria }{ }^{1} \\
\text { Ansiedad / Depresión / Insomnio } \\
\text { Dolor Crónico / Catastrofismo }\end{array}$ & \\
\hline $\begin{array}{l}102 \text { pacientes } \\
1^{\circ} \text { a } 3^{\circ} \text { Día Postoperatorioº } \\
\text { EN O a 10, reposo y movimiento } \\
\text { Localización } \\
\text { Analgesia de rescate / Efectos adversos } \\
\text { Descanso nocturno } \\
\text { Expectativas / Satisfacción }\end{array}$ & $\begin{array}{l}\text { 11 Excluidos } \\
\text { 5: ventilación mecánica > } 24 \mathrm{~h} \\
\text { 5: delirio postoperatorio } \\
\text { 1: no circulación extracorpórea }\end{array}$ \\
\hline $\begin{array}{l}97 \text { pacientes } \\
\text { Encuesta Postoperatoria }{ }^{3} \text { DCPQ } \\
\text { Dolor: intensidad / frecuencia / limitación funcional } \\
\text { Consumo de analgésicos } \\
\text { Descanso nocturno / Catastrofismo }\end{array}$ & $\begin{array}{l}5 \text { Excluidos } \\
\text { No localizados telefónicamente }\end{array}$ \\
\hline
\end{tabular}

${ }^{1}$ Adaptado del Brief Pain Inventory BPI-SF. ${ }^{2}$ Adaptada del cuestionario de la American Society of Pain. ${ }^{3}$ Test de Lattinen. EN: Escala Numérica del dolor. DCPQ: Dolor Crónico Postquirúrgico.

Fig. 1. Diagrama de flujo. 
TABLA I

DATOS DEMOGRÁFICOS Y DE COMORBILIDAD

\begin{tabular}{|c|c|c|c|c|}
\hline \multicolumn{2}{|c|}{$\begin{array}{l}\text { Datos demográficos y comorbilidad } \\
\text { Media (DS) o porcentaje (frecuencia] }\end{array}$} & $\begin{array}{c}A C \\
(n=52)\end{array}$ & $\begin{array}{c}\text { PCA } \\
(n=50)\end{array}$ & $A C$ vs. PCA \\
\hline Sexo & $72 \%$ hombres (74) & $77 \%(40)$ & $68 \%$ [34] & $p=0,183$ \\
\hline Edad & $65,55(11,01)$ & $64,67(10.1)$ & 66,43 (11.9) & $p=0,399$ \\
\hline IMC & $28,15(4,17)$ & $28,7(4,7)$ & $27,6(3,5)$ & $p=0,052$ \\
\hline ASA & $\begin{array}{c}3(0,64) \\
\% \text { ASA } \geq 4=18 \%(19)\end{array}$ & $\begin{array}{c}\% \text { ASA } \geq 4=17 \\
\% \text { (9) }\end{array}$ & $\begin{array}{c}\% \text { ASA } \geq 4=20 \% \\
\text { (10) }\end{array}$ & $p=0,445$ \\
\hline NYHA & $\begin{array}{c}2[0,76] \\
\% \mathrm{NYHA} \geq 3=17 \%(17)\end{array}$ & $\begin{array}{c}\% \text { NYHA } \geq 3=19 \\
\%(10)\end{array}$ & $\begin{array}{c}\% \text { NYHA } \geq 3=14 \\
\%(7)\end{array}$ & $p=0,603$ \\
\hline Euroscore I (logarítmico) & $4,5(3,14)$ & & & \\
\hline Euroscore II & $\begin{array}{c}2,4(1,85) \\
\text { \% Euroscore } \| \geq 3=28 \% \\
(29)\end{array}$ & $\begin{array}{c}\% \text { Euroscore } \| \geq \\
3=21 \%[11]\end{array}$ & $\begin{array}{l}\text { \%Euroscore } \| \geq 3 \\
\quad=36 \%(18)\end{array}$ & $p=0,124$ \\
\hline $\begin{array}{l}\text { Dolor crónico } \\
\text { preoperatorio }\end{array}$ & 25 \% [26] & $23 \%$ (12) & $28 \%(14)$ & $p=0,650$ \\
\hline Ansiedad & $17 \%(17)$ & $21 \%(11)$ & $12 \%(6)$ & $p=0,214$ \\
\hline Insomnio & 28 \% (29) & $29 \%$ (15) & $28 \%(14)$ & $p=0,444$ \\
\hline Catastrofismo & $17 \%(17)$ & $17 \%(9)$ & $16 \%[8]$ & $p=0,859$ \\
\hline Comorbilidad & $63 \%[64]$ & $58 \%$ (30) & $68 \%$ (34) & $p=0,303$ \\
\hline \multicolumn{5}{|c|}{ Tipo de comorbilidad (n) } \\
\hline Insuficiencia Renal & \multicolumn{4}{|l|}{14} \\
\hline Diabetes Mellitus & \multicolumn{4}{|l|}{25} \\
\hline Dolor Crónico & \multicolumn{4}{|l|}{28} \\
\hline S. Ansioso-Depresivo & \multicolumn{4}{|l|}{31} \\
\hline Cirugía Mayor previa & \multicolumn{4}{|l|}{5} \\
\hline C. Cardiaca previa & \multicolumn{4}{|l|}{1} \\
\hline Consumo de opioides & \multicolumn{4}{|l|}{4} \\
\hline Adicto a drogas & \multicolumn{4}{|l|}{2} \\
\hline
\end{tabular}

tratamiento analgésico recibido. No hubo diferencias entre los dos grupos de pacientes (PCA $98 \%$ vs. AC $92 \%, p=0,183$ ).

Se registraron un $7 \%$ de eventos adversos en relación con la analgesia, 3 pacientes con náuseas/vómitos, 3 con síndrome confusional y un íleo postoperatorio. Sin diferencias significativas entre los dos grupos, (PCA $4 \%$ vs. AC 10\%, $p=0$,262).

No hubo diferencias en la estancia media (EM) en UCl (2,2 vs. 2,2 días) o en el hospital, (PCA 10,5 vs. AC 10,7 días).

No se registraron diferencias en la intensidad del dolor entre los pacientes con remifentanilo (84\%) o con fentanilo (16\%) $(p=0,201)$.

La incidencia de DCPQ fue de un $39 \%$ a los tres meses, $11 \%$ a los 6 meses y $3 \%$ al año. El porcentaje de pacientes con DCPQ intenso fue de 9, 1 y $1 \%$, respectivamente. Sin diferencias significativas entre los dos grupos de pacientes a los tres meses (PCA $44 \%$ vs. AC $37 \%, p=0,533$ ).

A los tres meses, un $2 \%$ de los pacientes con DCPQ consumió regularmente analgésicos y tuvo una limitación funcional importante, y un $14 \%$ presentó insomnio y perfil catastrofista. Al año, ningún paciente tomaba regularmente analgésicos ni tenía ninguna limitación funcional por el dolor, un $3 \%$ tomaba hipnóticos y $1 \%$ presentaba un perfil catastrofista.

La intensidad del DAP se correlacionó negativamente con la edad, sobre todo en el 2. ${ }^{\circ}$ DPO $(r=-0,311$, $p=0,01$ ). Se registró una correlación positiva entre el dolor dinámico, el insomnio, la ansiedad y la presencia de dolor preoperatorios $(r=0,287, p=0,03)$. El perfil del paciente catastrofista se relacionó con mayor intensidad de DAP, sobre todo en el 3. er DPO $(r=0,285$, $p=0,04)$. No se encontró correlación entre el DAP y el sexo ni con el tipo y duración de la cirugía. 
TABLA II

DATOS INTRAOPERATORIOS, TIPO DE ANESTESIA Y TIPO DE ANALGESIA POSTOPERATORIA

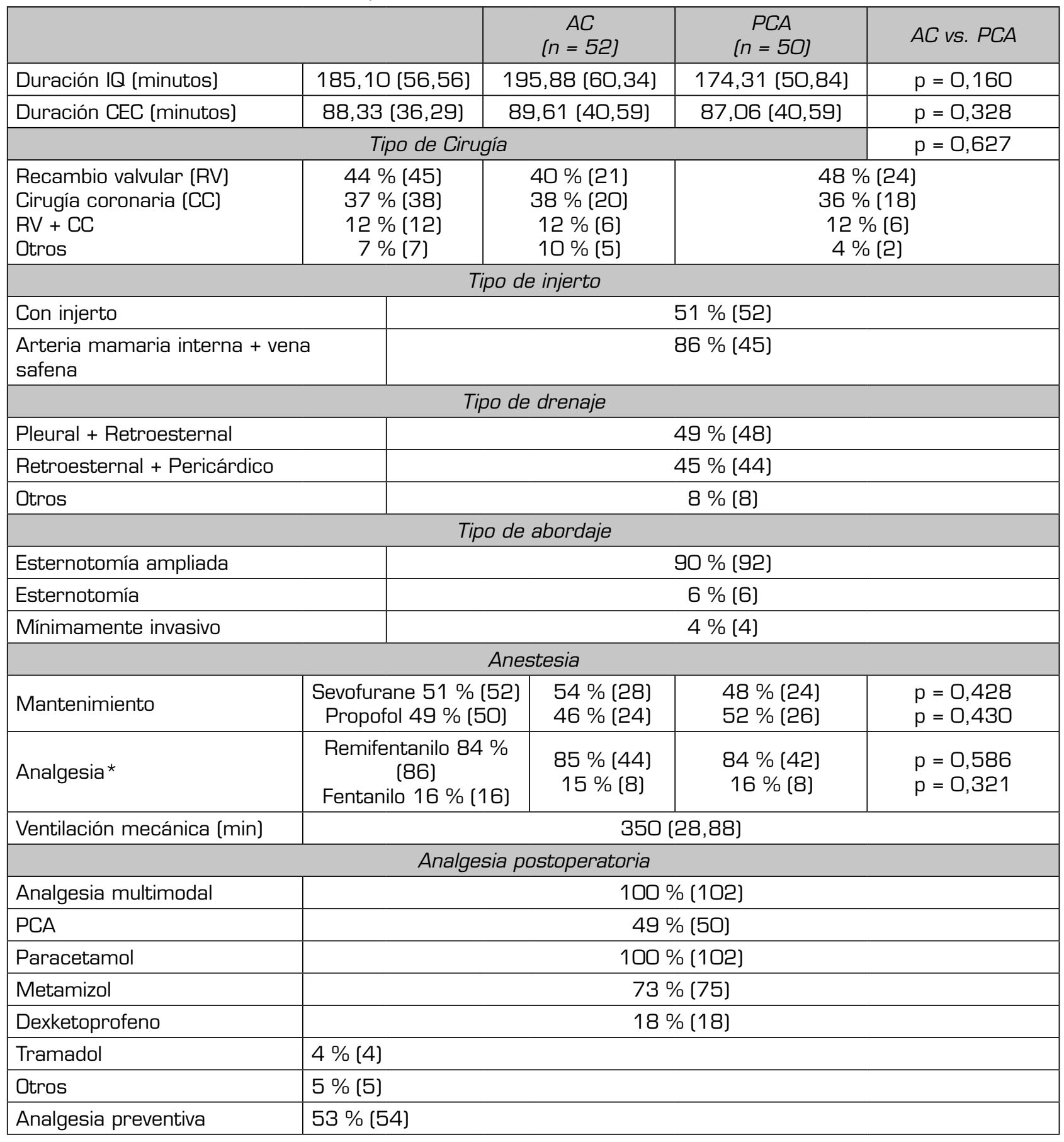

AC: Analgesia convencional. PCA: analgesia controlada por el paciente.

$\left[{ }^{*}\right.$ ] dosis de remifentanilo $<0,2 \mu / \mathrm{kg} / \mathrm{min}$ y de fentanilo $<15 \mu / \mathrm{kg}$.

Analgesia preventiva: dexketoprofeno (50 mg) o metamizol (2 g), paracetamol (1 g), dexametasona (8 mg) y tramadol (100 $\mathrm{mg}$ ) o morfina (5 mg).

\section{DISCUSIÓN}

Los datos de este estudio muestran una mediana de dolor en reposo inferior a EN 3 para todos los pacien- tes. Sin embargo, un $20 \%$ de los pacientes tuvieron dolor moderado y un $9 \%$ dolor intenso en reposo. En movimiento se registró un $34 \%$ de dolor moderado y un $38 \%$ de dolor intenso. Estos datos confirman el 

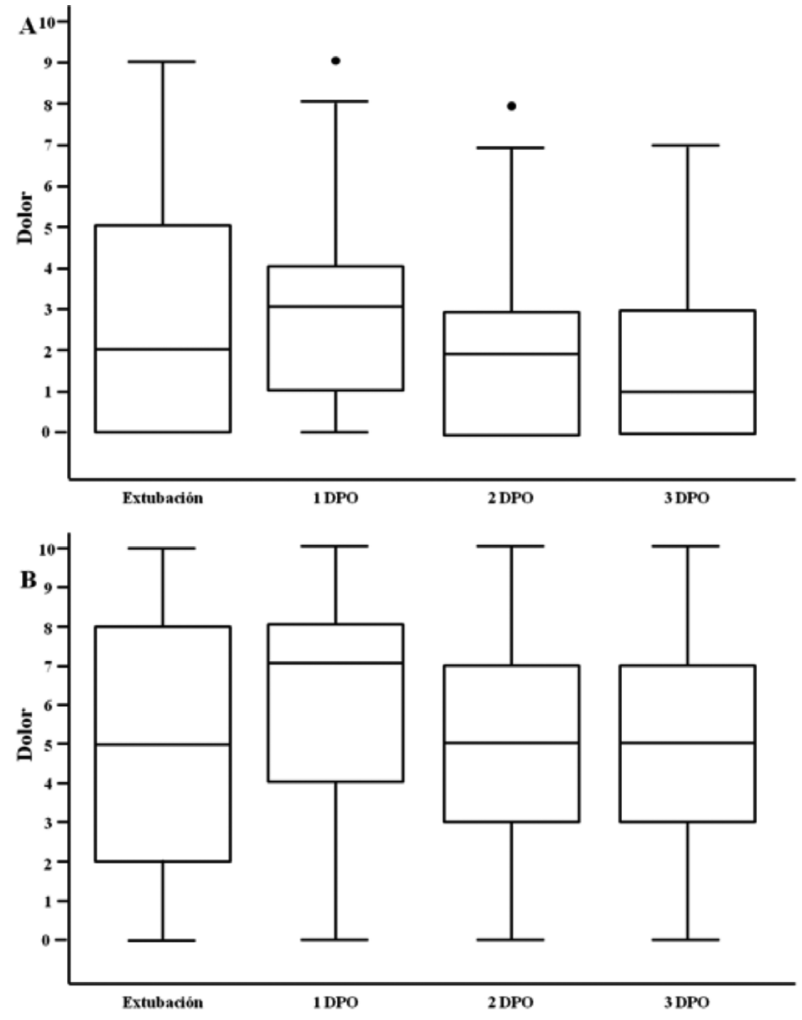

Fig. 2. Evolución del DAP en reposo (A) y en movimiento $(B)$ desde la extubación hasta el $3^{\circ}$ día postoperatorio (DPO), medido con la EN (O a 10). (Datos expresados en mediana, rango intercuartil y máximo-mínimo-outliers].

hecho de que las medias de dolor en un procedimiento quirúrgico no describen adecuadamente la efectividad de los tratamientos analgésicos para todos los pacientes.

El dolor dinámico fue siempre de moderado a intenso. El control del dolor al movimiento en CC es indispensable para garantizar una movilización y fisioterapia respiratoria precoz, un bloqueo de estímulos adrenérgicos, un confort adecuado del paciente y minimizar la posibilidad de presentar dolor crónico postquirúrgico (DCPQ). No obstante, los resultados de la práctica quirúrgica actual (14) cuestionan el hecho de que el control del DAP se base solo en el objetivo de obtener un valor en la $\mathrm{EN}<3$. Se ha demostrado que el inicio precoz de la deambulación, de la fisioterapia o de la rehabilitación, son factibles con un nivel moderado de dolor. Pretender un dolor dinámico con EN < 3 sin tener en cuenta la repercusión funcional, puede asociarse a inmovilidad del paciente o a efectos secundarios de los analgésicos que retrasen la recuperación. Se plantea el concepto de analgesia óptima postoperatoria (15), que se basa en optimizar el bienestar del paciente, acelerar la recuperación funcional y minimizar efectos secundarios.

La localización del dolor en la esternotomía en el $89 \%$ de los pacientes, coincide con otros estudios [16]
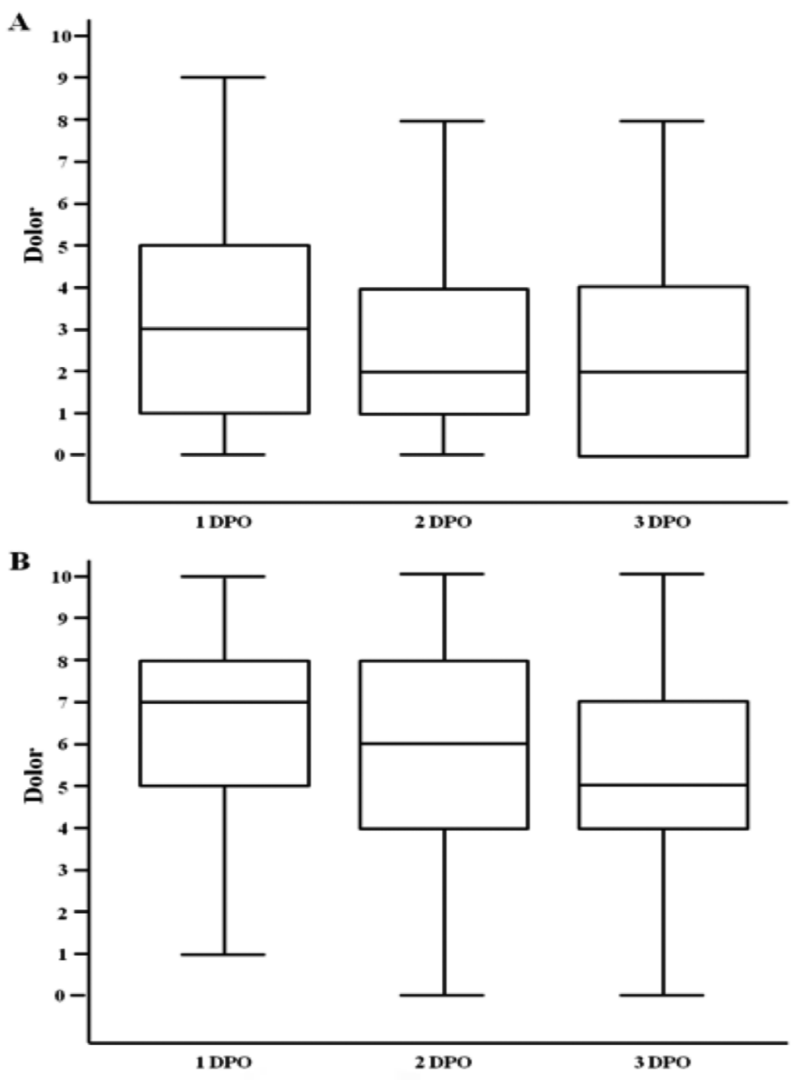

Fig. 3. Evolución del DAP máximo en reposo (A) y en movimiento $(B)$ en los primeros 3 días postoperatorios (DPO), medido con la EN (O a 10) (datos expresados en mediana, rango intercuartil y máximo-mínimo).

en los 3 primeros DPO, aunque se ha descrito dolor en el área de la safenectomía a partir del cuarto día, coincidiendo con una mayor deambulación.

No se registraron diferencias significativas en la intensidad del DAP entre los dos grupos de pacientes. El porcentaje de dolor en reposo de moderado a intenso (27\%) es inferior al 45-85\% registrado en otros estudios $(6,8)$. Esto indica que ambas pautas de analgesia fueron eficaces y que, en el grupo de la analgesia convencional, la enfermería actuó con la misma efectividad que los bolos administrados a demanda del paciente en el grupo PCA.

Los pacientes con PCA precisaron menos analgesia de rescate, menos dosis de morfina de rescate y pasaron más rápidamente a la analgesia vía oral.

La percepción del DAP presenta una gran variabilidad interindividual. Los factores que se han asociado a una mayor intensidad del DAP en CC son la edad menor a 65 años, el injerto de arteria mamaria interna, la ansiedad preoperatoria (6) y el catastrofismo (17). En este estudio, además de la menor edad, la ansiedad y un perfil catastrofista, la presencia de dolor preoperatorio y de insomnio, se asoció también a una mayor intensidad del dolor. Se han descrito consumos medios de morfina intravenosa de $50 \pm 15 \mathrm{mg}$ en los tres primeros días del postoperatorio [18]. Estos valores medios 


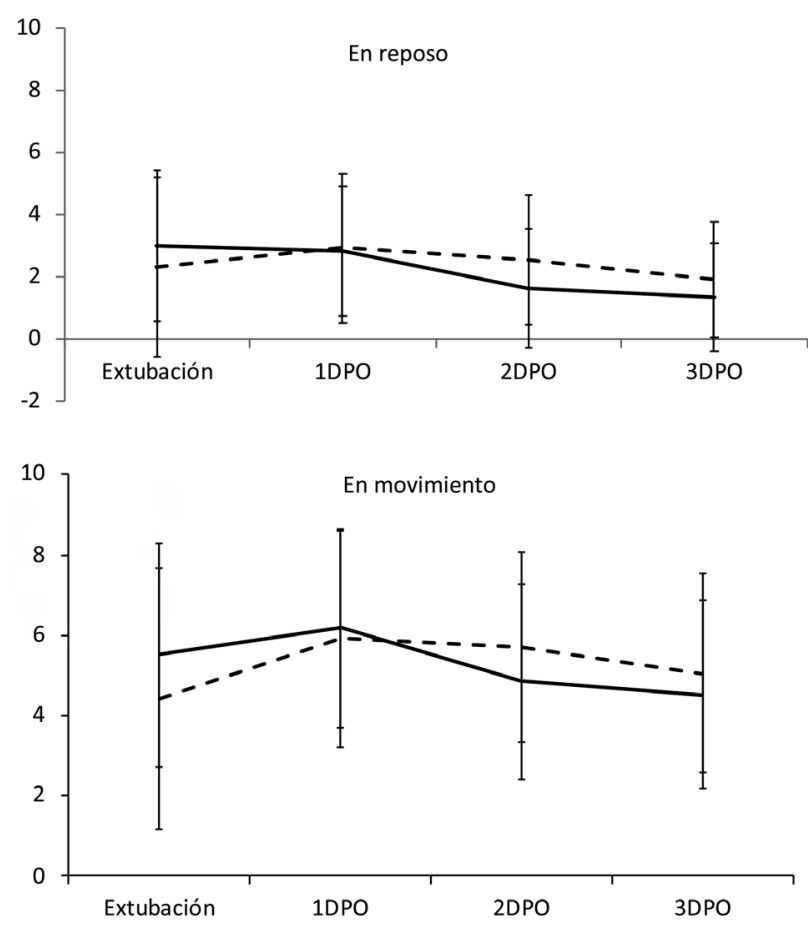

Fig. 4. Evolución de la EN del DAP en reposo y en movimiento desde la extubación hasta el 3.er día postoperatorio (DPO), en el grupo con analgesia convencional (AC) y con PCA. Datos expresados en media y DS $(p>0,05)$.

indican que los requerimientos de los pacientes pueden variar mucho. Se registran requerimientos de analgesia de rescate en un $65 \%$ de los pacientes (19). Este porcentaje es similar al $63 \%$ de los pacientes del grupo de AC que precisaron analgesia de rescate, y mayor que las demandas analgésicas de rescate del grupo PCA, (63 \% vs. $44 \%, p=0,0487$ ). Estos datos refuerzan la necesidad de pautar siempre analgesia de rescate, además de la pauta analgésica basal, para adecuar el tratamiento a cada paciente. Otros factores que pueden modificar las necesidades analgésicas y que, por tanto, hay que tener en cuenta al planificar la analgesia postoperatoria son: el consumo previo de opioides, la reintervención quirúrgica, la duración prolongada de la intervención, el síndrome ansioso-depresivo u otra intervención quirúrgica asociada.

La detección en el preoperatorio de estos grupos de pacientes con mayor riesgo de presentar dolor intenso en el postoperatorio, permitiría aplicar medidas terapéuticas específicas, como la PCA en modo continuo, la analgesia regional o los fármacos coadyuvantes que puedan potenciar los analgésicos.

Los opioides i.v. son los analgésicos más efectivos en el tratamiento dolor de moderado a intenso. No obstante, hoy en día se aplican pautas de ahorro de opioides [opioid-sparing o low-opioid] para minimizar sus potenciales efectos adversos y contribuir a una recuperación postoperatoria precoz. El modo de administración más efectivo es el controlado por el paciente (19-21) (PCA), que se ha demostrado que disminuye el consu- mo de morfina, controla mejor el dolor y se adapta a las necesidades individuales de cada paciente, además de disminuir las cargas de trabajo de enfermería. Sin embargo, en este estudio no registramos diferencias entre la PCA y la analgesia convencional con dosis a demanda administradas por la enfermería. En la revisión sistemática de Nachiyunde y cols. (21) se describen también diferentes resultados según los estudios. Este hecho se basa en la presencia de factores determinantes, como son la formación de la enfermería con respecto a la analgesia, la ratio enfermera/paciente y la existencia de protocolos analgésicos específicos [8]. Por otro lado, la efectividad de la PCA [22) es mayor en cirugías que presentan mayor intensidad de dolor en reposo, como la cirugía abdominal mayor abierta o la toracotomía.

No detectamos diferencias en la satisfacción del paciente, según el tipo de analgesia. Aunque el porcentaje de satisfacción fue superior en el grupo PCA, no alcanzó diferencias significativas, (PCA $98 \%$ vs. AC $92 \%, p=0,183$ ). Este es un indicador complejo, relacionado con las expectativas del paciente y también con las experiencias previas. Por ello, pueden darse tasas de satisfacción elevadas junto a un control deficiente del dolor.

Se ha demostrado que la analgesia multimodal disminuye el consumo de opioides y sus efectos secundarios (5). En este estudio, el $100 \%$ de los pacientes recibieron analgesia multimodal. Un $100 \%$ de paracetamol, un $73 \%$ de metamizol, además de la morfina en PCA o en dosis de rescate.

En CCA existe controversia sobre el uso de los AINE [20]. Se han demostrado seguros en pacientes menores de 70 años y con función renal preoperatoria normal. Su uso durante un corto periodo de tiempo, en estos pacientes, no se ha asociado a mayor mortalidad, infarto de miocardio, disfunción renal o sangrado gastrointestinal $(19,20)$.

Se han descrito otras opciones analgésicas efectivas desde un punto de vista multimodal $[23,24]$, sin que haya suficiente evidencia para su recomendación generalizada. Se ha utilizado la analgesia epidural (25), la pregabalina (26), la oxicodona vía oral (27), la dexmedetomidina o la analgesia regional (bloqueo paravertebral [28], bloqueo intercostal o bloqueo paraesternal [29]).

Se han registrado incidencias de DCPQ en CC entre un 11 y $46 \%$ de los pacientes, aunque solo de un 2 a un $10 \%$ de los mismos presentan dolor severo $[24,29)$. La incidencia registrada en este estudio de un $3 \%$ de DCPQ al año es inferior a otras series, como la de Hoogd y cols. (30) de un $19 \%$ o la de Lahtinen y cols. [31] de un $16 \%$. Todos coinciden en que el porcentaje de pacientes con dolor intenso y limitación funcional al año es bajo. En nuestro caso fue de un $1 \%$, sin diferencias entre los dos grupos de pacientes. Hay que destacar la heterogeneidad de los estudios y de los métodos de evaluación del DCPQ. Así, por ejemplo, Setälä y cols. [32] señalaban la discordancia entre los datos registrados por un cuestionario telefónico [38\%) y por la exploración clínica (15\%).

El $84 \%$ de los pacientes recibieron una perfusión de remifentanilo con dosis inferiores $a<0,2 \mu / \mathrm{kg} / \mathrm{min}$. El uso de remifentanilo se ha asociado a mayor intensidad de DAP y a mayor incidencia de DCPQ. No obstante, los datos son controvertidos. En el estudio de Hoogd 
y cols. (33) se registra más dolor y más consumo de opioides postoperatorios junto a mayor incidencia de DCPQ a los 3 meses, pero no al año. Esto puede explicar los resultados contradictorios de los distintos trabajos. Nuestros resultados no muestran relación entre el uso de remifentanilo o fentanilo y el DAP o el DCPQ. No obstante, el grupo con fentanilo fue muy bajo (16\%).

No hubo diferencias en la estancia media en la UCl o en el hospital. Sin embargo, la validez de la EM como indicador de efectividad analgésica es limitada, porque depende de otros factores como la variabilidad de la práctica clínica o las distintas formas de organización hospitalaria.

Las limitaciones de este estudio se derivan de su carácter observacional y de la evaluación antes-después de la aplicación del protocolo analgésico de PCA. A pesar de la homogeneidad de ambos grupos de pacientes, no se pueden descartar sesgos por la falta de aleatorización.

\section{CONCLUSIONES}

La PCA con opioides a demanda y analgesia multimodal es una alternativa efectiva después de una cirugía cardiaca. Se obtiene un buen control del dolor, un paso rápido a la analgesia vía oral, sin incrementar efectos adversos y precisando menos analgesia de res- cate administrada por enfermería. El tipo de analgesia no influyó en la estancia media ni en la incidencia de $\mathrm{DCPQ}$. Se registra un porcentaje de pacientes con dolor moderado e intenso que precisarían protocolos analgésicos específicos para poder mejorar los resultados postoperatorios.

\section{AGRADECIMIENTOS}

A la sección de anestesiólogos de Cirugía Cardiaca, al Dr. J. I. Sáez de Ibarra-Sánchez, Jefe de Servicio y al equipo de cirujanos del Servicio de Cirugía Cardiaca, a la Dra. L. Vidal-Bonet del Servicio de Cardiología, a la enfermería de la Unidad de Recuperación Postanestésica y a la enfermería de la Unidad de Cuidados Intensivos, cuya colaboración ha sido imprescindible para la realización de este trabajo.

La presente investigación no ha recibido ayudas específicas provenientes de agencias del sector público, sector comercial o entidades sin ánimo de lucro.

\section{CONFLICTO DE INTERESES}

Los autores declaran no tener ningún conflicto de intereses.

\section{ANEXO 1 \\ Dolor postoperatorio en cirugía cardiaca. 2017 Encuesta preoperatoria}

Paciente: $\quad \mathrm{N}^{0} \mathrm{H}^{\mathrm{a}}$

Fecha: / /

1. ¿Padece insomnio?

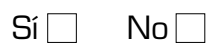

2. ¿Toma medicamentos para dormir?

$$
\text { Sí } \square \quad \text { No } \square
$$

3. ¿Sufre ansiedad/depresión?

$$
\text { Sí } \square \quad \text { No } \square
$$

4. ¿Toma medicamentos para la ansiedad o la depresión?

$$
\text { Sí } \square \quad \text { No } \square
$$

5. ¿Padece dolor crónico?

$$
\text { Sí } \square \quad \text { No } \square
$$

6. ¿Tiene dolor en este momento?

$$
\text { Sí } \square \quad \text { No } \square
$$

7. ¿Cuánto dolor? De $\mathrm{O}$ a 10 
Si padece dolor crónico:

8. ¿El dolor le ha afectado en los siguientes aspectos de la vida, durante la última semana? Actividades en general:

Estado de ánimo:

Capacidad de caminar:

Trabajo habitual (incluye tareas domésticas) :

Relaciones personales:

Sueño:

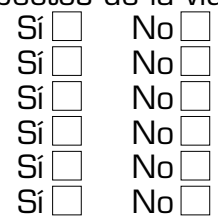

¿Está de acuerdo o se identifica con alguna de estas frases?

9. No dejo de pensar en lo mucho que me duele

$$
\text { Sí } \square \quad \text { No } \square
$$

10. No hay nada que pueda hacer para aliviar la intensidad del dolor

$$
\text { Sí } \square \quad \text { No } \square
$$

11. Temo que el dolor empeore

$$
\text { Sí } \square \quad \text { No } \square
$$

\section{ANEXO 2}

\begin{tabular}{|c|c|c|c|c|c|}
\hline & & Extubación & $1^{\circ} \mathrm{DPO}$ & $2^{\circ} D P O$ & $3^{\circ} \mathrm{DPO}$ \\
\hline \multicolumn{6}{|l|}{ En reposo } \\
\hline \multicolumn{6}{|l|}{ En movimiento } \\
\hline \multirow{3}{*}{$\begin{array}{l}\text { Localización } \\
\text { (máximo dolor] }\end{array}$} & Esternotomía & & & & \\
\hline & Músculo-esquelético & & & & \\
\hline & Drenajes & & & & \\
\hline \multicolumn{6}{|l|}{ En máximo reposo } \\
\hline \multicolumn{6}{|l|}{ En máximo movimiento } \\
\hline \multirow{2}{*}{ Analgesia de rescate } & Sí & & & & \\
\hline & No & & & & \\
\hline \multirow{2}{*}{ Descanso noc } & Sí & - & & & \\
\hline & No & - & & & \\
\hline
\end{tabular}

Encuesta de dolor postoperatorio CC - 2017

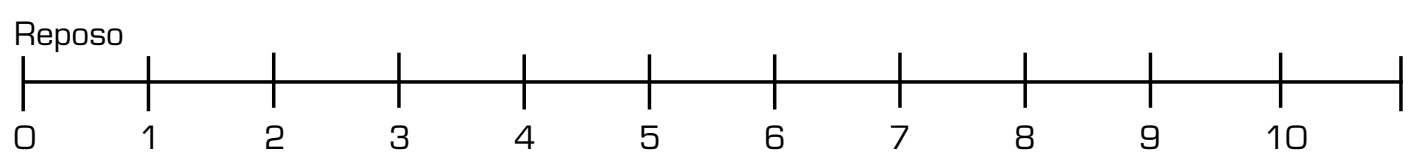

\section{Preguntas en el $3 .^{\circ}$ DPO}

Comparado con el dolor que esperaba, ¿cuánto dolor ha tenido después de esta operación?

$\square$ Mucho más de lo esperado $\square$ Algo más de lo esperado $\square$ Como esperaba $\square$ Un poco menos de lo esperado $\square$ Mucho menos de lo esperado

¿Cree usted que puede aliviarse el dolor tras una operación quirúrgica como la suya?

$\square$ Poco $\square$ Algo $\square$ Mucho $\square$ Alivio total

¿Cuál es su grado de satisfacción con el tratamiento del dolor?

$\square$ Muy insatisfecho $\square$ Insatisfecho $\square$ Satisfecho $\square$ Muy satisfecho 


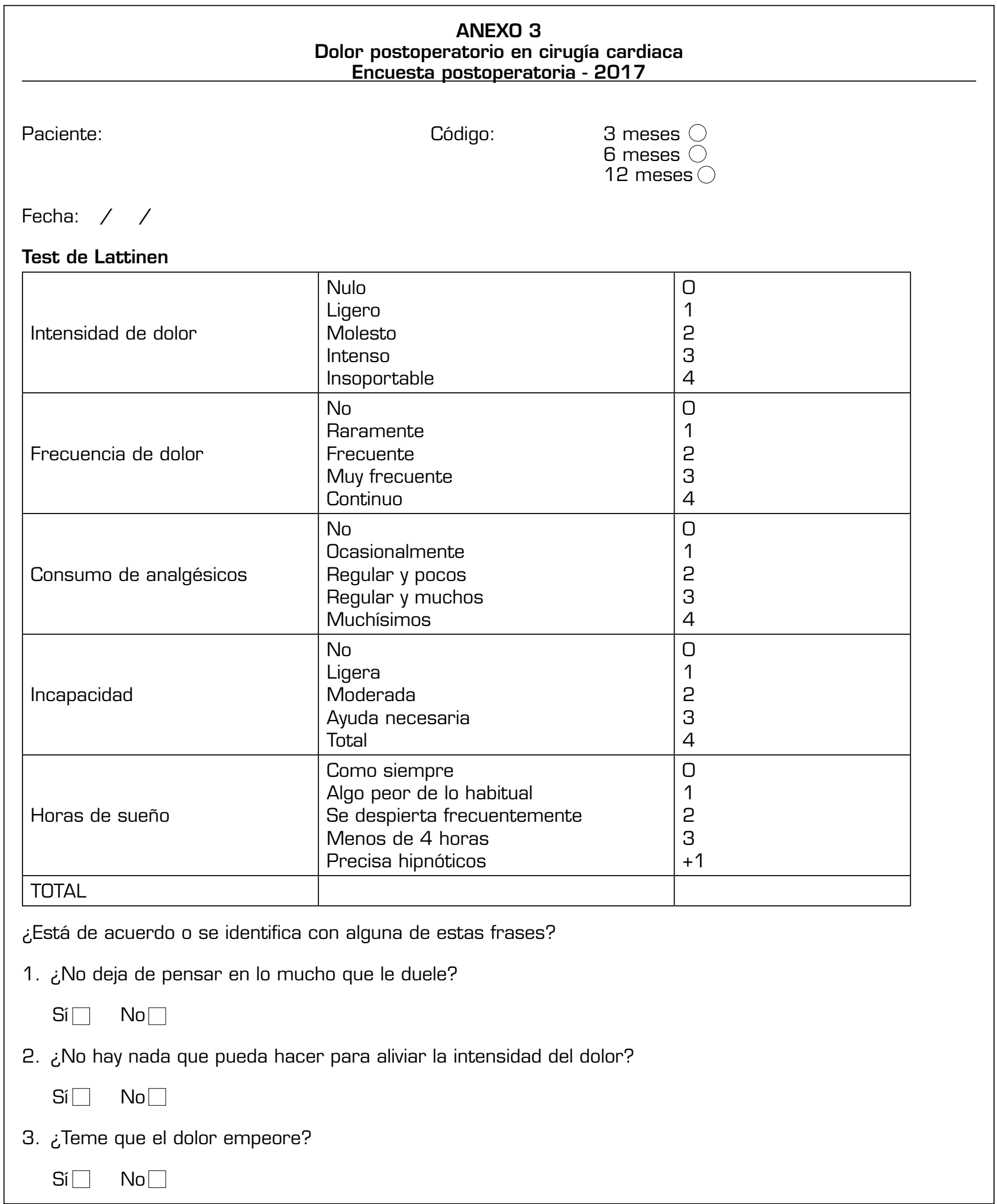




\section{BIBLIOGRAFÍA}

1. Kehlet H. Postoperative pain, analgesia, and recoverybedfellows that cannot be ignored. Pain. 2018;159(Suppl 1):S11-S16. DOI: 10.1097/j.pain.0000000000001243.

2. Rawal N. Current issues in postoperative pain management. Eur J Anaesthesiol. 2016;33(3):160-71. DOI: 10.1097/ EJA.0000000000000366.

3. Gewandter JS, Dworkin RH, Turk DC, Farrar JT, Fillingim RB, Gilron I, et al. Research design considerations for chronic pain prevention clinical trials: IMMPACT recommendations. Pain. 2015;156(7):1184-97. DOI: 10.1097/j. pain.0000000000000191.

4. Peláez R, Hortal FJ, Riesgo M. Tratamiento del dolor postoperatorio en cirugía cardíaca. Rev Esp Anestesiol Reanim. 2002;49(9):474-84.

5. Rafiq S, Steinbrüchel DA, Wanscher MJ, Andersen LW, Navne A, Lilleoer NB, et al. Multimodal analgesia versus traditional opiate based analgesia after cardiac surgery, a randomized controlled trial. J Cardiothorac Surg. 2014;9:52 DOI: 10.1186/1749-8090-9-52.

6. Navarro MA, Irigoyen Ml, De Carlos V, Martínez A, Elizondo A, Indurain S, et al. Evaluación del dolor postoperatorio agudo tras cirugía cardiaca. Enferm Intensiva. 2011;22(4):150-9. DOI: 10.1016/j.enfi.2011.04.002.

7. Keawnantawat P, Thanasilp S, Preechawong S. Translation and Validation of the Thai Version of a Modified Brief Pain Inventory: A Concise Instrument for Pain Assessment in Postoperative Cardiac Surgery. Pain Pract. 2017;17(6):76373. DOl: 10.1111/papr.12524.

8. Bjørnnes AK, Rustøen T, Lie I, Watt-Watson J, Leegaard M. Pain characteristics and analgesic intake before and following cardiac surgery. Eur J Cardiovasc Nurs. 2016;15(1):47-54. DOI: $10.1177 / 1474515114550441$

9. Bignami E, Castella A, Pota V, Saglietti F, Scognamiglio A, Trumello C, et al. Perioperative pain management in cardiac surgery: a systematic review. Minerva Anestesiol. 2018;84(4):488-503. DOI: 10.23736/S03759393.17.12142-5.

10. Badia X, Muriel C, Gracia A, Núñez-Olarte JM, Perulero N, Gálvez R, et al; Grupo Vesbpi. Validation of the Spanish version of the Brief Pain Inventory in patients with oncological pain. Med Clin (Barc). 2003;120(2):52-9. DOI: 10.1016/ s0025-7753(03)73601-x.

11. Díez-Alvarez E, Arrospide A, Mar J, Cuesta M, Martínez Mdel C, Beitia E, et al. Assessment of acute postoperative pain. Rev Calid Asist. 2009;24(5):215-21. DOl: 10.1016/j. cali.2008.12.001.

12. González-Escalada JR, Camba A, Muriel C, Rodriguez M, Contreras D, Barutell C. Validation of the Lattinen Index for the assessment of chronic pain patients. Rev Soc Esp Dolor. 2012;19(4):181-8.

13. Doğan Bakı E, Kavrut Ozturk N, Ayoğlu RU, Emmiler M, Karsı B, Uzel H. Effects of Parasternal Block on Acute and Chronic Pain in Patients Undergoing Coronary Artery Surgery. Semin Cardiothorac Vasc Anesth. 2016;20(3):205-12. DOI: 10.1177/1089253215576756.

14. Regenbogen SE, Mullard AJ, Peters N, Brooks S, Englesbe MJ, Campbell DA Jr, et al. Hospital Analgesia Practices and Patient-reported Pain After Colorectal Resection. Ann Surg. 2016;264(6):1044-50. DOI: 10.1097/ SLA.0000000000001541.

15. McEvoy MD, Scott MJ, Gordon DB, Grant SA, Thacker JKM, Wu CL, et al. American Society for Enhanced Recovery (ASER) and Perioperative Quality Initiative (POQI) joint consen- sus statement on optimal analgesia within an enhanced recovery pathway for colorectal surgery: part 1-from the preoperative period to PACU. Perioper Med (Lond). 2017;6:8. DOI: 10.1186/s13741-017-0064-5.

16. Roca J, Valero R, Gomar C. Pain locations in the postoperative period after cardiac surgery: Chronology of pain and response to treatment. Rev Esp Anestesiol Reanim. 2017;64(7):391400. DOI: 10.1016/j.redar.2017.01.002.

17. Khan RS, Skapinakis P, Ahmed K, Stefanou DC, Ashrafian $H$, Darzi A, et al. The association between preoperative pain catastrophizing and postoperative pain intensity in cardiac surgery patients. Pain Med 2012;13(6):820-7. DOI: 10.1111/j.1526-4637.2012.01386.x.

18. Ruetzler K, Blome CJ, Nabecker S, Makarova N, Fischer H, Rinoesl $\mathrm{H}$, et al. A randomised trial of oral versus intravenous opioids for treatment of pain after cardiac surgery. J Anesth. 2014;28(4):580-6. DOI: 10.1007/s00540-013-1770-x.

19. Mota FA, Marcolan JF, Pereira MH, Milanez AM, Dallan LA, Diccini S. Comparison study of two different patient-controlled anesthesia regiments after cardiac surgery. Rev Bras Cir Cardiovasc. 2010;25(1):38-44. DOI: 10.1590/s010276382010000100011

20. Bainbridge D, Cheng DC, Martin JE, Novick R; EvidenceBased Perioperative Clinical Outcomes Research (EPiCOR) Group. NSAID-analgesia, pain control and morbidity in cardiothoracic surgery. Can J Anaesth. 2006;53(1):46-59. DOI: 10.1007/BFO3021527.

21. Nachiyunde B, Lam L. The efficacy of different modes of analgesia in postoperative pain management and early mobilization in postoperative cardiac surgical patients: A systematic review. Ann Card Anaesth. 2018;21(4):363-70. DOI: 10.4103/aca.ACA_186_17.

22. Peng L, Ren L, Qin P, Su M. The impact of patient-controlled analgesia on prognosis of patients receiving major abdominal surgery. Minerva Anestesiol. 2016;82(8):827-38.

23. Ziyaeifard M, Azarfarin R, Golzari SE. A Review of Current Analgesic Techniques in Cardiac Surgery. Is Epidural Worth it? J Cardiovasc Thorac Res. 2014;6(3):133-40. DOI: 10.15171/jcvtr.2014.001.

24. Rovira I. Analgesia postoperatoria para cirugía cardiaca mínimamente invasiva: ¿cuál es la técnica ideal? Rev Esp Anestesiol Reanim. 2012;59(9):467-9. DOI: 10.1016/j. redar.2012.07.010.

25. Jakobsen CJ. High thoracic epidural in cardiac anesthesia: a review. Semin Cardiothorac Vasc Anesth. 2015;19(1):3848. DOI: 10.1177/1089253214548764.

26. Joshi SS, Jagadeesh AM. Efficacy of perioperative pregabalin in acute and chronic post-operative pain after off-pump coronary artery bypass surgery: a randomized, double-blind placebo controlled trial. Ann Card Anaesth. 2013;16(3):180-5. DOI: 10.4103/0971-9784.114239.

27. Ruetzler K, Blome CJ, Nabecker S, Makarova N, Fischer H, Rinoesl $\mathrm{H}$, et al. A randomised trial of oral versus intravenous opioids for treatment of pain after cardiac surgery. J Anesth. 2014;28(4):580-6. DOl: 10.1007/s00540-013-1770-x.

28. Ho AM, Karmakar MK, Ng SK, Wan S, Ng CS, Wong RH, et al. Local anaesthetic toxicity after bilateral thoracic paravertebral block in patients undergoing coronary artery bypass surgery. Anaesth Intensive Care. 2016;44(5):615-9. DOI: 10.1177/0310057X1604400502.

29. Gjeilo KH, Stenseth R, Klepstad P. Risk Factors and Early Pharmacological Interventions to Prevent Chronic Postsurgical Pain Following Cardiac Surgery. Am J Cardiovasc Drugs 2014;14(5):335-42. DOI: 10.1007/s40256-014-0083-2. 
30. de Hoogd S, Valkenburg AJ, van Dongen EPA, Daeter EJ, van Rosmalen J, Dahan A, et al. Short- and long-term impact of remifentanil on thermal detection and pain thresholds after cardiac surgery: A randomised controlled trial. Eur J Anaesthesiol. 2019;36(1):32-9. DOI: 10.1097/ EJA.0000000000000887.

31. Lahtinen P, Kokki H, Hynynen M. Pain after cardiac surgery: A prospective cohort study of 1-year incidence and intensity. Anesthesiology. 2006;105(4):794-800. DOI: 10.1097/00000542-200610000-00026.
32. Setälä P, Kalliomäki ML, Järvelä K, Huhtala H, Sisto T, Puolakka P. Postoperative hyperalgesia does not predict persistent post-sternotomy pain; observational study based on clinical examination. Acta Anaesthesiol Scand. 2016;60(4):520-8. DOI: 10.1111/aas.12659.

33. de Hoogd S, Ahlers SJGM, van Dongen EPA, van de Garde EMW, Daeter EJ, Dahan A, et al. Randomized controlled trial on the influence of intraoperative remifentanil versus fentanyl on acute and chronic pain after cardiac surgery. Pain Pract. 2018;18:443-51. DOI: 10.1111/papr.12615. 